\title{
ABordagem holística do TERMO PESSOA EM UM ESTUdo EMPÍRICO: UMA ANALISE CRÍTICA
}

\author{
David Lopes Neto ${ }^{1}$ \\ Lorita Marlena Freitag Pagliuca ${ }^{2}$
}

Lopes D Neto, Pagliuca LMF. Abordagem holística do termo pessoa em um estudo empírico: uma análise crítica. Rev Latinoam Enfermagem 2002 novembro-dezembro; 10(6):825-30.

O estudo analisa a semântica e a estrutura do termo pessoa (mulheres com HIV), mediante abordagem holística fundamentada no Modelo de Adaptação de Roy (Veritivity) e na fenomenologia de Heidegger (Dasein). Pesquisa extraída da literatura das Ciências Enfermagem e Filosofia, através da técnica de documentação indireta, com utilização do modelo de análise de teoria de Chinn \& Kramer, através do qual foram analisados os seguintes aspectos: objetividade, simplicidade, generalidade, acessibilidade e importância dos conceitos de pessoa e holismo no estudo empírico "Nutritional adaptation of women living with HIV: a pilot study", publicado na revista Holistic Nursing Practice, em 1997. Criticamente, a interpretação do termo pessoa se deu pela compreensão do ser enquanto um ser ôntico-ontológico, como forma de percepção verdadeira do sentido do ser (pessoa), como uma estrutura universal vivente em um mundo antropológico e social, ao qual mulheres com HIV podem se adaptar ao novo estilo de vida e receber atenção integral da enfermagem, principalmente no que concerne ao aspecto nutricional.

DESCRITORES: teoria de enfermagem, modelos de enfermagem

\section{HOLISTIC APPROACH OF THE TERM “PERSON" IN AN EMPIRICAL STUDY: A CRITICAL ANALYSis}

The study analyzes the semantic and the structure of the term "person" (women with HIV), by means of an holistic approach based on the Roy Adaptation Model (Veritivity) and on Heidegger's Phenomenology (Dasein). This research was extracted of Nursing Science and Philosophy of Science literature, through the technique of indirect documentation, using the model of theoretical analysis developed by Chinn \& Kramer, through which the following aspects were analyzed: objectivity, simplicity, generality, accessibility and importance of person and holism concepts in the empirical study Nutritional adaptation of women living with HIV: a pilot study, published in the journal Holistic Nursing Practice, in 1997. Critically, the interpretation of the term "person" was a result of the understanding of "being" as an ontical-ontological being, as a form of true perception of the sense of being (person), as an alive universal structure in an anthropological and social world, in which women with HIV can adapt to the new lifestyle and receive integral care provided by nursing, mainly with respect to the nutritional aspect.

\section{ABORdAJE HOLÍSTICO DEL TÉRMINO PERSONA EN UN ESTUdIO EMPIRICO: UN ANALISIS CRÍTICO}

El estudio analiza la semántica y la estructura del término: persona (mujeres con HIV), mediante el abordaje holitico basado en el Modelo de Adaptación de Roy (Veritivity) y en la fenomenología de Heidegger (Dasein). Investigación extraída de la literatura de las Ciencias de enfermería y filosofía, a través de la técnica de documentacion indirecta, con la utilización del modelo de análisis de teoria de Chinn \& Kramer a través del cual se analizaron los siguientes aspectos: la objetividad, simplicidad, generalidad, accesibilidad y importancia de los conceptos de persona y holismo en el estudio empírico Nutritional adaptation of women living with HIV: a pilot study, publicado en la revista Holistic Nursing Practice, en 1997. Críticamente, la interpretación del término persona se dio por la comprensión de ser como ontico-ontológico, como forma de verdadera percepción del sentido de ser (persona), como una estructura universal viva en un mundo antropológico y social al que las mujeres con HIV se pueden adaptar al nuevo estilo de vida y recibir cuidado integral de la enfermera, principalmente en lo que se relaciona con el aspecto nutricional.

DESCRIPTORES: teoría de enfermería, modelos de enfermería

\footnotetext{
${ }^{1}$ Doutor em Enfermagem, Professor da Escola de Enfermagem de Manaus da Universidade do Amazonas, e-mail: dln@baynet.com.br; ${ }^{2}$ Doutor
} em Enfermagem, Professor Titular do DENF/FFOE/Universidade Federal do Ceará 
INTRODUÇÃO

O termo holismo vem sendo empregado na enfermagem como abordagem que espelha as realidades complexas dos seres humanos no cosmo. Nesta virada de século e de milênio, um modo de pensar energiza o pensamento humano, como um fio condutor de um novo paradigma. Nesse contexto transcendental, a abordagem holística emerge como técnica holográfica - modelo de interação dos elementos que compõem o todo e as partes em que se reflete uma realidade tridimensionalizada ${ }^{(1)}$.

Envolvendo a Ciência da Enfermagem nesse orbe de constituição interativa do ser, que contempla o sentido do viver humano em um plano biopsicossocial-espiritual e da completa harmonia e equilíbrio desse ser com o ambiente que o circunda, o seu propósito filosófico é uno, ao considerar o traço estrutural holístico como abordagem pertinente às suas teorias, exemplificado no Modelo de Adaptação de Roy.

Neste estudo, desenvolvemos uma análise crítica do conceito essencial à natureza da Enfermagem - pessoa, em um estudo empírico, dentro de uma abordagem holística, mediante os pressupostos teóricos de Roy e da fenomenologia existencial de Heidegger.

\section{MATERIAL E MÉTODO}

Pesquisa extraída da literatura da Enfermagem e da Filosofia, através da técnica de documentação indireta, com a finalidade de tentar explicar o pensamento acerca da temática selecionada. A pesquisa foi desenvolvida em cumprimento às partes teórica e prática da disciplina Análise crítica de teorias de enfermagem, do Programa de Pós-Graduação em Enfermagem, em nível de Doutorado em Enfermagem, da Universidade Federal do Ceará, ministrada no segundo semestre de 1999. O estudo analisa o continuum semântico e estrutural holístico no conceito de pessoa, mediante análise crítica de cunho teóricofilosófico, baseada na fenomenologia existencial de Martin Heidegger (Dasein) e no Modelo de Adaptação de Roy (veritivity), considerando-se que são aspectos concernentes ao estudo empírico, com utilização do modelo de análise de teoria de teóricas da enfermagem ${ }^{(2)}$, através do qual são analisados os seguintes aspectos: objetividade, simplicidade, generalidade, acessibilidade e importância dos conceitos de pessoa e holismo no estudo empírico "Nutritional adaptation of women living with HIV: a pilot study"(3).

\section{ABORDAGEM HOLÍSTICA NO MODELO DE ADAPTAÇÃO DE ROY}

Os modelos de enfermagem estão baseados em suposições científicas e filosóficas e, por conseguinte, o desenvolvimento do conhecimento, nessa disciplina, reflete e avança as suposições, cotidianamente, quando teóricas de enfermagem identificam convicções e valores ao construírem seus modelos teóricos.

O Modelo de Adaptação de Roy foi contextualizado e espelhado nas suposições da Teoria Geral dos Sistemas de von Bertalanffy e da Teoria do Nível de Adaptação de Helson, as quais contribuíram para a construção do marco filosófico pautado em uma visão humanística e holística (veritivity), haja vista que Roy vê a pessoa funcionando com partes mutuamente dependentes que agem em unidade por algum propósito decorrente de um sistema integrado.

O Modelo de Adaptação de Roy é um modelo conceitual, permitindo que a pessoa seja vista como um sistema composto por vertentes de ordens biopsicossocial, as quais requerem respostas para a adaptação da pessoa aos estímulos ambientais. Na conformidade desse modelo, a pessoa é vista como um sistema que tem a capacidade de criar mudanças para se adaptar ao ambiente, onde a habilidade e a capacidade de criação dessas mudanças é o nível de adaptação da pessoa ${ }^{(4)}$.

\section{CLARIDADE E CONSISTÊNCIA SEMÂNTICA E ESTRUTURAL DO CONCEITO DE PESSOA DE ROY NA ABORDAGEM HOLÍSTICA}

A Enfermagem, ao longo dos anos, vem incluindo na sua literatura a abordagem holística, de forma explícita e objetiva. Estudos descreveram que a abordagem holística na Ciência de Enfermagem, a partir da década de 1980, está cada vez mais sendo evidenciada, saindo do oculto, do não revelado. Nas décadas anteriores, através de outros termos, o holismo era abordado nos modelos teóricos de enfermagem como estado biopsicossocial do ser humano ou função integralizadora da totalidade do indivíduo, como parte dos conceitos que 
formulavam o pensamento teórico da Enfermagem ${ }^{(5)}$.

A enfermagem holística abraça toda prática de enfermagem, cuja premissa é a de cuidar da pessoa inteira, reconhecendo que há duas visões relativas ao holismo: aquela em que o holismo estuda e entende os interrelacionamentos das dimensões biopsicossocialespirituais da pessoa e reconhece que o todo é maior que a soma de suas partes; aquela em que o holismo entende o indivíduo como um todo integrado, interagindo com outrem através de ambientes internos e externos. Segundo a Association's Standards of Holistic Nursing Practice, dos Estados Unidos, conceitos holísticos incorporam um equilíbrio sensível entre arte e ciência, habilidades intuitivas e analíticas, habilidade e interconexões de corpo, mente e espírito $^{(6)}$.

Em relação aos conceitos chaves do modelo de Roy, um neologismo criado por ela, veritivity, definido como um princípio da natureza humana que afirma uma intencionalidade comum da existência humana, expressa, ao nosso ver, a natureza do termo holismo, sob a forma conceitual abstrata, mas se objetiva no cuidar de enfermagem.

\section{SER HOLÍSTICO NA PERSPECTIVA FENOMENOLÓGICA DE HEIDEGGER}

A interpretação do termo pessoa, sob a ótica da fenomenologia existencial de Martin Heidegger, inicia-se pela compreensão do ser ôntico-ontológico, como forma de entendimento verdadeiro do sentido do ser, para nós expressado, etimologicamente, neste estudo, como pessoa. De acordo com essa corrente, busca-se perceber pessoa na sua estrutura universal, enquanto ser vivente. Todavia, o homem só poderá se compreender se tiver percepção da totalidade de si próprio e da sua real existência $^{(6)}$. Nesse sentido, a essência do pensamento heideggeriano é a preocupação com o sentido do ser existente - o Dasein.

Martin Heidegger, no seu livro Ser e Tempo, tenta mostrar a maneira de ser da pessoa pela inspeção reflexiva e explicativa do que ele chamou de ser especial. O filósofo enfatiza o holismo ao relacionar o ser - pessoa - com o cosmos na identificação que faz do ser-no-mundo, referindo-se a um fenômeno de unidade. Para caracterizar unidade, totalidade em uma vertente ôntica, ele trabalha com o conceito de mundaneidade, como um lugar onde os seres dos entes e das coisas se interrelacionam, dãose mutuamente. $\mathrm{Na}$ vertente ontológica, essa caracterização é descrita pelo significado da estrutura vivencial momentânea da pessoa no mundo antropológico/ cosmológico e a pessoa não é uma coisa, algo substancial ou objeto; é unidade de corpo, alma e espírito ${ }^{(7)}$.

Na enfermagem, a pessoa é parte de um ambiente harmonioso e relacional, o qual envolve diferentes pessoas - enfermeiro, clientes, familiares e outros profissionais. Essa integração, pessoa-pessoa e pessoa-mundo espelha a abordagem holística tão evidente nas raízes existenciais da enfermagem teórica, onde corpo, mente e espírito são inseparáveis e partes constituintes da pessoa que, submetida à cotidianidade, é um ser-no-mundo, assim como tão bem elucidou Heidegger.

\section{ANÁLISE ETIMOLÓGICA E SEMÂNTICA DO CONCEITO DE PESSOA}

Etimologicamente, pessoa, personlichkeit (alemão), person (inglês), personne (francês), persona (italiano), deriva do latim persona, definida como papel, caráter, personagem, indivíduo a quem se atribui direitos e obrigações ${ }^{(8)}$.

A pessoa é um corpo que se manifesta como um fenômeno da sua pessoa moral (ser individual que possui características que lhe permitem participar da sociedade intelectual e moral dos espíritos: consciência de si, razão, quer dizer, capacidade de distinguir o verdadeiro e o falso, o bem e o mal) enquanto exprime o seu caráter ${ }^{(9)}$.

Criticamente, a utilização do termo pessoa tem duas origens: a primeira, por uma conotação da idéia estóica do papel desempenhado pelo homem no mundo e, a segunda, pelo emprego da palavra pela teologia no que se refere às controvérsias de que - "Deus é pessoal?"(9), haja vista que essa idéia de um Deus pessoal não está relacionada diretamente à natureza Divina, mas à pessoa no mundo, compreendendo-se que Cristo é Deus natureza divina e Jesus é homem e Filho do Homem e, Jesus Cristo a forma encarnada da ação de Deus no mundo.

A pessoa é um sistema adaptativo, descrito como um conjunto de partes que funcionam como unidades pertencentes a um todo que constitui a natureza humana $^{(10)}$.

Todas as teorias e modelos de enfermagem 
incluem idéias sobre a natureza dos seres humanos. $\mathrm{O}$ componente filosófico mais consistente da idéia pessoa é a dimensão totalidade ou holismo. A natureza de holismo, como um conceito, é de difícil definição, dadas as perspectivas filosóficas ocidentais tradicionais, baseadas na idéia reducionista de interpretação do holismo, no qual o todo é igual às partes, através da soma das partes, enquanto que a verdade do termo é a harmonia entre as partes que compõem o todo, assim como as partes que compõem a pessoa, como um ser biopiscossocialespiritual. Roy enfatiza que a pessoa é um sistema humano que tem a capacidade de se ajustar efetivamente às mudanças e aos efeitos causais, advindos do ambiente $^{(4)}$.

\section{SÍNTESE DO ESTUDO EMPÍRICO}

Foi analisado o estudo de Orsi AJ, Grady C, Tax A, Mccorkle, R. "Nutritional adaptation of women living with HIV: a pilot study", traduzido como: "Adaptação nutricional de mulheres que vivem com HIV: um estudo piloto". O artigo examinou as respostas imunológicas e nutricionais de um pequeno grupo de 13 mulheres infectadas por HIV, tendo como marco referencial o Modelo de Adaptação de Roy, o qual, segundo as autoras, pode servir de guia para pesquisas com mulheres portadoras de HIV.

Os resultados indicaram que mulheres com um nível de estabilidade da doença podem não ter problemas com a adaptação nutricional e que enfermeiras juntamente com as pacientes podem ser vigilantes constantes sobre o monitoramento de peso e, assim, identificarem precocemente os principais problemas relacionados com essa variável e promoverem estratégias de respostas adaptativas, mediante uma prática de enfermagem holística, que consista em assistir a cliente biopsicossocial e espiritualmente. Em conclusão ao estudo, as enfermeiras sugeriram que outros estudos longitudinais, usando o Modelo de Adaptação de Roy com largas amostras de mulheres e de variáveis sóciodemográficas, são necessários para determinar os padrões de respostas que acometem as mulheres portadoras de HIV.

\section{ESTRUTURA DE ANÁLISE DE TEORIAS/ MODELOS DE ENFERMAGEM}

Nas teorias e modelos de enfermagem, são feitas suposições sobre pessoas e seus comportamentos, pelo que a enfermeira, ao escrever suas intenções, necessita explorar, com clareza e consistência, os conceitos abstraídos das teorias e modelos de enfermagem, para predizer a sua real intervenção. Investigadoras da enfermagem ${ }^{(11)}$ explicam que as enfermeiras que aplicam, na prática, as teorias e modelos de enfermagem precisam estar atentas aos aspectos que envolvem o desenvolvimento do processo de enfermagem. Para que isso ocorra, elas devem conhecer as estruturas dos modelos de avaliação existentes. Neste estudo, são utilizados para análise os aspectos do modelo analítico de teóricas da enfermagem ${ }^{(2)}$, formados a partir de cinco questões propiciadoras de análise crítica: objetividade, simplicidade, generalidade, acessibilidade e importância do conceito.

\section{ANÁLISE DO ARTIGO}

Objetividade do conceito

A claridade semântica e estrutural do conceito de pessoa está presente no estudo desde a sua introdução. Já no início, o texto é apresentado pelas autoras, recorrendo às suas experiências, conduzindo a interpretações claras e concisas do seu objeto conceitual: pessoas (mulheres com HIV), o que lhes serviu de ajuda para confrontar seus resultados com a literatura, referendando uma clareza evidente do termo pessoa no estudo em referência.

O fenômeno (mulheres com HIV) é caracterizado, em todo o estudo, pela disposição do sentido da palavra mulheres, pessoas com capacidades biopsicossocialespirituais. Assim, podemos considerar que a objetividade está na manifestação da idéia em si, proveniente da verdade atual dessas mulheres, considerando a possibilidade que elas têm de poder ter uma adaptação nutricional e, a partir daí, relacionarem-se de maneira equilibrada com o mundo e com as coisas quer as circundam, caracterizando objetivamente sua existência como ser-no-mundo.

\section{Simplicidade do conceito}

O estudo descreve com simplicidade, o elemento conceitual pessoa (mulheres com HIV), como seres 
humanos constituídos de mente-corpo e espírito, no ambiente clínico da enfermagem prática. Para essa descrição, as teóricas valeram-se do Modelo de Adaptação de Roy e assumiram a afirmação de que mulheres com HIV são seres adaptáveis e, também, na concepção filosófica heideggeriana de que pessoas são sempre círculos hermenêuticos de entendimento ${ }^{(3)}$. Entendimento é um processo reflexivo de mudança entre uma impressão global da experiência, ou seja, mais que um enfocar das partes das experiências vividas pela pessoa holisticamente, uma visão da totalidade das mulheres portadoras de $\mathrm{HIV}^{(12)}$

\section{Generalidade do conceito}

O conceito de pessoa encontra-se definido em todas as teorias de enfermagem, em virtude da exigência da própria filosofia da enfermagem, cuidar de pessoas. A generalidade conceitual do termo pessoa na abordagem holística é descrita no estudo, por considerar esse a essência conceitual do Modelo de Adaptação de Roy, construída a partir da amplitude da Teoria Geral dos Sistemas e do holismo presentes na pesquisa, quando as autoras sempre enfatizam Roy, como um modelo de cuidado estruturado para o entendimento holístico das clientes.

A forma conceitual com que o termo pessoa (mulheres com HIV) é apresentado no estudo é denotada nas diferentes situações ou campos de ação onde possa ser aplicado, quando referido às mulheres portadoras de HIV, o que expressa um sentido de generalização.

Em uma perspectiva epistemológica, trata-se de um elemento de conhecimento que exprime os princípios e as proposições da enfermagem. A generalização do termo pessoa está representada, no texto, na seguinte frase: "o propósito do estudo-piloto foi examinar o estado imunológico e nutricional de um pequeno grupo de mulheres infectadas por HIV e utilizar o Modelo de Adaptação de Roy, como ferramenta holística para entender as mudanças no estilo de vida de mulheres portadoras de HIV". Nesse texto, a idéia central dá a representatividade de um objeto geral (mulheres igual a pessoas do sexo feminino), em um sentido de extensão do termo a todo um grupo de pessoas que são portadoras do vírus HIV (mulheres com HIV).

A generalização é uma "operação extensiva a toda uma classe ... aquilo que foi observado em um número limitado de indivíduos ou casos singulares que pertencem a esta classe"(8).

Acessibilidade do conceito

A identificação do termo pessoa foi facilitada pelo engajamento das autoras na enfermagem clínica e teórica - professoras, pesquisadoras e enfermeiras assistenciais, fato esse que, possivelmente, reforçou o processo de entendimento decorrente da validação do fenômeno observado, o fato empírico.

O estudo vai ao encontro da análise empírica tradicionalmente usada na pesquisa em enfermagem. Enfermeiras teóricas, ao descreverem a importância do pluralismo metodológico, na pesquisa em enfermagem, afirmaram que há quatro tipos de pesquisas mais exploradas pela enfermagem: a análise empírica, a teoria fundamentada, a hermenêutica heideggeriana e a pesquisaação ${ }^{(13)}$. A consistência estrutural do estudo, em conseqüência da fundamentação do conceito de pessoa (mulheres com HIV), através da observação empírica paralela à corrente fenomenológica de interpretação do ser, revela a acessibilidade conceitual do indicador empírico.

Há, portanto, lógica na colocação do objeto de pensamento no texto, o que o torna compreensível. A função dos caracteres que definem o termo pessoa, permeia por todo o texto, expressando, de forma acessível a idéia das autoras. Elas, em linguagem de fácil compreensão, descrevem os elementos lógicos correspondentes à idéia central do estudo, afirmando que mulheres com HIV, com nível satisfatório de estabilidade da doença, podem ter significativa adaptação nutricional.

Importância do conceito

No estudo, o termo pessoa é analisado com base na abordagem da totalidade do ser, extrapolando a dimensão biológica, para alcançar dimensões humanísticas, holísticas e antropológicas. Com base no pensamento de Sister Callista Roy, o estudo considera que pessoa é mais que um ser biológico, enquanto que a enfermagem é mais que uma prática biologicista - é uma ciência que incorporou uma visão da vida humana no mundo.

O mérito do termo pessoa, compreendido em uma abordagem holística, está nas formas pelas quais as 
autoras do estudo empírico o definiram, precisando com muita propriedade o sentido do termo, sustentando, com grande valor, sobre uma base teórica da enfermagem.

\section{CONSIDERAÇÕES FINAIS}

Há uma facilidade analógica e crítica apresentada quanto ao sentido da palavra holismo, no Modelo de Adaptação de Roy, quando a expressão pessoa é tomada na linguagem filosófica, considerando, em especial, a complexidade do termo em toda a acepção da palavra, ou na força da totalidade do termo, ao designar o modo próprio de ser de cada ser humano.

A análise do estudo conduziu à precisão e à integração das informações contidas no texto, no qual variáveis sóciodemográficas integradas descreveram os padrões de conhecimento da enfermagem prática. $\mathrm{O}$ termo pessoa foi conceitualizado, seguindo os postulados teóricos de Roy, representando o todo que é o ser humano.

Teóricas de enfermagem concordam que o recipiente do cuidado de enfermagem é reflexo de um sistema adaptativo holístico, sendo o termo holístico, originário da condição e das suposições filosófico-

\section{REFERÊNCIAS BIBLIOGRÁFICAS}

1. Lopes D Neto, Nóbrega MML. Holismo nos modelos teóricos de enfermagem. Rev Bras Enfermagem 1999, abr/ jun; 52(2):233-42.

2. Chinn PL, Kramer MK. Theory and Nursing: a systematic approach. St. Louis: Mosby; 1995.

3. Orsi AJ, Grady C, Tax A, Mccorkle R. Nutritional adaptation of women living with HIV: a pilot study. Holist Nurs Pract 1997; 12(1):71-6.

4. Roy SC. Theotist's response to Strengthening the Roy Adaptation Model through Conceptual Clarification. Nurs Sci Q 1990; 3(2):64-6.

5. Sarkis JM, Skoner MM. An analysis of concept of holism in nursing literature. Holistic Nurs Pract 1987; 2(1):61-9.

6. Dossey BM, Kee L, Guzetta CE, Kolkmeier LG. Holistic Nursing: a handbook for practice. Maryland: Aspen; 1995.

7. Heidegger M. Ser e Tempo. 4a ed. Petrópolis: Vozes; 1993.

8. Rocha R. Minidicionário. São Paulo: Scipione; 1996.

9. Lalande A. Vocabulário técnico e crítico de filosofia. $2^{\mathrm{a}}$ ed. São Paulo: Martins Fontes; 1996.

10. Roy SC, Andrews HA. The Roy Adaptation Model: the definitive statement. Connecticut: Appleton \& Lange; 1991.

11. Aggleton $P$, Chalmers $H$. Nursing models and the nursing process. London: MaCMilllan; 1986. humanísticas, pertencente à idéia de que as funções do sistema humano são um todo e são mais que as meras somas das partes, e que é expressado, com bastante propriedade, pelas autoras do estudo empírico ${ }^{(9)}$.

Em síntese, na abordagem holística é enfatizada a conexão e a ligação entre as partes e o todo, mediante a consciência humana das partes (corpo-mente-espíritoenfermagem-cliente-família, etc) e no pensamento heideggeriano as mulheres portadoras de HIV são seresno-mundo em busca de um viver de acordo com as suas maneiras de estarem no mundo.

A pessoa cônscia de si torna-se mais consciente das partes das outras pessoas e ambas terão liberdades de escolha para responder aos estímulos ${ }^{(13)}$. No estudo, é retratada essa resposta holística quando as autoras descrevem habilidades e técnicas de como intervir no estado nutricional alterado da pessoa portadora de HIV. A resposta holística da enfermagem está em assistir as pessoas, conscientizando-as sobre o viver a vida. Por fim, modelos de avaliação ajudam a olhar, com olhos críticos, as teorias e os modelos de enfermagem. Qualquer estudo que utilize teorias e modelos de enfermagem estarão, por conseguinte, contribuindo para o desenvolvimento da enfermagem teórica.

12. Walters AJ. Being a clinical nurse consultant: a hermeneutic phenomenological reflection. Int. Nurs Pract 1996; 2(1):5

13. Pietroni P. Viver holístico. São Paulo: Summus; 1988. 